\title{
La familia y las visitas en las unidades de
} cuidado intensivo

Diana Marcela Achury, Master of Nursing (Cardiovascular Health), Universidad Nacional. Assistant Professor, Nursing Faculty, Pontificia Universidad Javeriana, Bogotá, Colombia.

Luisa Fernanda Achury Beltrán. Critical Care Nurse Specialist, Pontificia Universidad Javeriana; Intensive Care Nurse, Hospital Universitario San Ignacio; Instructor Professor, Pontificia Universidad Javeriana, Bogotá, Colombia.

Ángela Marcela Ramírez Cárdenas. Critical Care Nurse Specialist, Pontificia Universidad Javeriana. Intensive Care Unit Manager, Clínica VIP Cafesalud Medicina Prepagada, Bogotá, Colombia.

\section{PALABRAS CLAVE: enfermería familia unidad cuidado intensivo visitas *}

E-mail: dianaachury@yahoo.es

\section{ENGLISH}

Family visiting in intensive care

Key words

Family, intensive care unit, nursing, visiting

Summary

- A patient's admission to the intensive care unit causes an imbalance for the whole family. Therefore, nurses and other healthcare professionals should focus caring on assessing and managing not only the patient's needs, but the family's too.

- Visiting is a vital tool that enables social, emotional, and informational needs to be met.

- In daily practice, there is a lack of clarity around visiting practices and policies, the nurse's professional role, and how visiting impacts upon the critically ill patient's recovery. The aim of this article is to provide information that enables nurses to make decisions about visiting and develop guidelines that enhance family satisfaction and impact positively on the patient's physical, emotional, and social recovery.

\section{RESUMEN}

- La hospitalización de un paciente en la Unidad de Cuidado Intensivo (UCI), ocasiona un desequilibrio para todo el núcleo familiar, razón por la cual el personal de salud, en particular el profesional de enfermería debe dirigir su cuidado a la valoración y manejo de las necesidades no sólo del paciente sino también de la familia.

- La visita se convierte entonces en una herramienta fundamental que permite satisfacer la demanda de necesidades de tipo social, emocional, de información y acompañamiento.

- En la práctica diaria no existe una claridad del tipo de visitas, las orientaciones, el rol del profesional de enfermería y el impacto de estas en la recuperación del paciente crítico.

- Este artículo busca proporcionarles a los profesionales de enfermería fundamentos que les permita comprender y aplicar lineamientos de las visitas soportados en la satisfacción de la familia, recuperación física, emocional y social del paciente.

\section{INTRODUCCIÓN}

A lo largo del tiempo la familia ha desempeñado un papel decisivo en el cuidado de los pacientes, pues es evidente que una mayor presencia de la familia también implicaría una participación más activa de estos en los cuidados e incluso en las decisiones que se toman respecto a ellos. Por otra parte, la atención a los familiares de nuestros pacientes es una pieza más de nuestros cuidados que en muchos casos queda olvidada por diferentes circunstancias como la realización de intervenciones de enfermería que exigen toda nuestra atención en el cuidado directo del paciente, la carga laboral y las tareas administrativas que se asumen en el cotidiano desempeño de nuestra labor reduciendo tiempo importante y esencial de acompañamiento y asistencia a los familiares del paciente, en el marco de un cuidado holístico.

De igual manera, es incuestionable que la atención más global del paciente de cuidados intensivos, requiere de la atención de sus necesidades afectivas y sociales, por lo tanto, la presencia más activa de la familia en sus cuidados (Fernández 2006). Para dar cumplimiento a la incorporación de la familia como cuidadora del paciente critico y objeto de cuidado por parte de los profesionales de enfermería, se describen a continuación algunos conceptos que permiten conocer el origen, tipo de visitas, factores influyentes, sus efectos y el papel del profesional de enfermería en la aplicación de lineamientos de las visitas soportados en la satisfacción de la familia, recuperación física, emocional y social del paciente.

\section{ORIGEN E HISTORIA DE LAS VISITAS}

Las visitas se originaron en 1965 cuando se dio lugar a la creación a las unidades de Cuidado Intensivo en Estados Unidos, siendo una característica importante de ellas, la limitación de ingreso a los familiares en periodos cortos de tiempo, puesto que se afirmaba que las visitas generaban efectos negativos en la salud y estado del paciente crítico por la complejidad de sus condiciones físicas, manejo y tratamientos (Titler \& Walsh 1992).

Por otro lado, se consideraba la familia como un componente poco relevante del entorno del paciente, concepto que ha cambiado con el transcurrir del tiempo, en donde se ha demostrado que los familiares pueden contribuir de forma significativa con la consecución de una adecuada evolución en el proceso de recuperación del paciente; 
sin embargo en Colombia este concepto es poco aplicado en la práctica actual, en donde la unidad de cuidado intensivo sigue siendo un espacio cerrado y regulado por los profesionales, esto significa que "los de dentro" (pacientes y profesionales de la salud) mantienen poco contacto (físico, emocional y de comunicación) con "los de fuera" (familiares/allegados de los pacientes) y en donde las políticas de visitas son restrictivas y estáticas (Zaforteza et al. 2004)

\section{NECESIDADES DEL PACIENTE Y LA FAMILIA}

El ingreso a la unidad de UCl altera el normal funcionamiento del círculo familiar, genera una serie de necesidades en el paciente y por ende en su familia, de manera que estas deben ser valoradas e identificadas oportunamente para poder ser satisfechas. Al revisar la literatura se encuentra que las principales necesidades de los familiares de los pacientes ingresados en $\mathrm{UCl}$ son: información, comunicación, personalización, seguridad, espirituales-religiosas y compartir la mayor parte del tiempo posible con su familiar; los familiares necesitan relacionarse con otras personas para vencer la sensación de soledad que están viviendo en la $\mathrm{UCl}$, necesitan confiar en el equipo de salud. (Henneman \& Cardin 2002; Livesay et al. 2005)

En la familia se ha encontrado que el ingreso de sus familiares a la $\mathrm{UCl}$ puede producir alteraciones psicológicas y sociales, en donde ellos experimentan niveles elevados de ansiedad y depresión, lo cual podría incrementar el riesgo de la falta de atención de sus propias necesidades, dificultades para tomar decisiones o solucionar problemas, todo esto, relacionado con la forma de aparición, gravedad y duración del proceso de la enfermedad en la unidad de cuidado intensivo (Garrouste-Orgeas et al. 2008)

Existen tres tipos de necesidades familiares: las de tipo social, práctico y emocional. Las de tipo social están expresadas en términos del contacto y mantenimiento de una proximidad con el paciente, las necesidades de tipo emocional hacen referencia a la esperanza, la tranquilidad y la expresión de sentimientos y las de tipo práctico se clasifican a su vez en no materiales (el horario de visitas flexible, la información obtenida del estado de salud y del entorno del paciente, las orientaciones sobre las acciones que deben realizar los familiares para contribuir al cuidado y la seguridad del paciente) y materiales (disponibilidad de espacios adecuados, ayuda económica en el caso que lo requiera) (Verhaeghe et al. 2005)

La satisfacción de estas necesidades se convierte entonces en la prioridad del equipo de salud durante la visita, sin embargo muchas de ellas no son identificadas ni tratadas por factores como tiempo, percepción de la familia y preparación y disponibilidad del personal. La literatura muestra que las necesidades practicas (no materiales) y emocionales son las más insatisfechas; en las necesidades practicas (no materiales) se encuentran la información y comunicación puesto que los familiares refieren tiempos de espera prolongados, errores en la continuidad y rapidez en la información proporcionada, lenguaje inapropiado utilizado por los profesionales, y una información incompleta, poco clara y oportuna sobre el estado de salud, dispositivos de soporte, procedimientos, tratamiento y formas de comunicación con el paciente en espacios incómodos y hostiles (Engström \& Söderberg 2004).

De todas estas necesidades, la información se convierte en un componente muy importante para los familiares y diversos estudios se han centrado en el análisis de diferentes modos de proporcionar información a los familiares de pacientes críticos, afirmando que un encuentro con los familiares puede reducir su agitación (GarrousteOrgeas et al. 2008) y ayudar a minimizar el posible conflicto entre el equipo de cuidados de la UCl y los familiares, además de proporcionar un espacio para educar y orientar a la familia.

Habitualmente, se cree que la información hace referencia exclusivamente al diagnóstico, pronóstico y tratamiento, por lo que se sostiene que la labor de informar es una tarea exclusiva de los médicos y no de enfermería. Es evidente el papel relevante del profesional de enfermería en la atención a familiares por la continua permanencia junto a los pacientes; dentro de un marco multidisciplinar de atención, se hace imprescindible la existencia de una adecuada coordinación de la misma y conocimiento de la información que transmite cada miembro del equipo, para mantener una uniformidad y evitar la incertidumbre (Simon et al. 1997)

\section{TIPOS DE VISITAS Y CONDICIONES DE LAS VISITAS}

Las características de la visitas están determinadas por: el tiempo de duración, número de familiares, forma de ingreso, infraestructura, tipo de información y la posibilidad de ingreso de niños visitantes y otros miembros allegados como amigos cercanos o familiares más lejanos a la Unidad.

A partir de estas características y del postulado en donde la principal necesidad tanto de la familia como del paciente en $\mathrm{UCl}$, es el compartir el mayor tiempo posible durante el periodo crítico de la hospitalización, se han clasificado las visitas en dos grupos: restrictivas y no restrictivas. Las vistas restrictivas establecen limitaciones de horario, cantidad y parentesco de los familiares, además de la imposición de un momento determinado para la obtención de la información acerca del estado de salud del paciente hospitalizado.

Las visitas no restrictivas se caracterizan por permitir a los familiares el ingreso a la unidad de cuidado intensivo en cualquier momento durante las 24 horas del día, con la frecuencia durante el tiempo que ellos deseen y permitiendo el ingreso de los niños a la unidad y de más de un familiar simultáneamente (Berti et al. 2007); de esta forma, la extensión del horario permite que el visitante pueda distribuir el tiempo con sus demás actividades, además de permitirle un mayor periodo de contacto con el paciente (Pérez et al. 2004).

El panorama internacional muestra que alrededor del $50 \%$ de las unidades de cuidado intensivo tienen visitas a puertas abiertas sin restricción del horario (Díaz et al. 2009). A nivel nacional existen muy pocas unidades con una real política de visitas abiertas siendo la tendencia el continuar bajo la implementación de las políticas restrictivas.

Esta situación evidencia la necesidad de reconocer que el tipo de visitas que se instaure en las unidades debe fundamentarse en aquella que de forma individualizada permita obtener el mayor beneficio en cuanto a la recuperación del paciente y la satisfacción de las necesidades de los familiares, de manera que se deben tener en cuenta las características anteriormente mencionadas para definir las visitas; la visita debe ser entonces el espacio para proporcionar la educación e información necesaria que permita la integración y ayuda del familiar en el cuidado del paciente crítico, teniendo en cuenta una constante evaluación y valoración de las respuestas que se presenten tanto en el paciente como en la familia para poder adoptar las acciones requeridas en caso que dichas reacciones sean negativas. Además las visitas con un mayor número de familiares deben ser aprovechadas para brindar la mayor información y educación posible evitando así desorganización en la visita y obteniendo los mayores beneficios para el paciente (Berti et al. 2007).

\section{FACTORES QUE INFLUYEN EN LA FLEXIBILIZACIÓN DE LAS VISITAS}

Existen unos factores que se asocian en el establecimiento del tipo de visitas con un grado de flexibilización, estos están relacionados con las políticas y los actores del proceso de cuidado en el paciente crítico. 
Respecto a las políticas se puede afirmar que tienen un impacto considerable sobre la satisfacción de las familias con el tratamiento y los pacientes, estas deben permitir la satisfacción de las necesidades sociales y emocionales del paciente y las de sus familias.

Las políticas en las unidades de cuidados intensivos de adultos han sido lentas en su cambio hacia la flexibilización de las visitas en comparación a las unidades de cuidados intensivos pediátricos, probablemente debido a que las necesidades relacionales del paciente pediátrico son más evidentes (Aronson et al. 2009).

Según la literatura el medio ambiente y los aspectos organizativos no influyen de manera significativa sobre las políticas de visitas, mientras que el tipo de unidad de cuidados intensivos (pediátrica o de adultos) y la experiencia y calificación de las enfermeras parecen estar directamente relacionadas con la libertad y flexibilidad en los horarios de visitas (Sims \& Miracle 2006). Adicionalmente se puede afirmar que las prácticas cotidianas de visitas no siempre se rigen a las normas o políticas institucionales, ya que están en su mayoría en manos de las personas que se encuentran a cargo de la unidad de cuidado intensivo, de manera que la liberalización de la visitas depende en mayor parte de las percepciones y creencias que tienen tanto los profesionales de la salud como los mismos familiares, pues hay quienes creen conveniente la restricción de la visita cuando lo estimase oportuno según la condición clínica del paciente o las intervenciones que se deban realizar (Berti et al. 2007; Lee et al. 2007). Hay opiniones a favor de mantener visitas restrictivas en la $\mathrm{UCl}$, justificándose en el descanso y la tranquilidad que requiere el paciente en estado crítico para lograr su recuperación (Livesay et al. 2005), pero se olvida que el paciente puede tener sensaciones de abandono y soledad durante su hospitalización, además de la incertidumbre que manifiestan los familiares al no tener contacto permanente con su ser querido; en otras opiniones se encuentra que el mayor contacto del paciente con personal que entra y sale constantemente de la unidad puede acarrear mayor número de infecciones aunque los estudios no han demostrado complicaciones a causa de estos (Fumagalli et al. 2006).

El factor humano como componente influyente de las políticas de la visitas, está relacionado con la tendencia a trabajar y actuar en conveniencia hacia los profesionales que laboran en las unidades de cuidado intensivo, probablemente por no tener el tiempo deseado para la atención de la familia, las suficientes capacidades de relación y comunicación y el conocimiento sobre cómo enfrentar las dudas y reacciones emocionales del paciente y su familia, lo cual ocasiona temor en el personal de la salud, de manera que se adopta la restricción de la visita como la mejor opción. Algunas veces el profesional de enfermería no se encuentra acostumbrado ni al ingreso ni a la permanencia constante del familiar en la $\mathrm{UCl}$ $y$ ve las visitas como intrusivas, dejando de lado todos los efectos positivos que estas traen consigo, no solo en términos de mejoría de la salud y planificación del cuidado, sino también en términos de calidad y satisfacción familiar (Fumagalli et al. 2006; Zaforteza et al. 2004). Sin embargo se encuentra mayor aceptación y una actitud más positiva por parte del personal de enfermería frente al personal médico ante una política liberalizada de la visitas en la $\mathrm{UCl}$, lo cual puede ser explicado por la formación académica integral de la enfermera, en donde se tiene como objetivo el cuidado no sólo al enfermo sino también a su familia entendiendo que las situaciones de crisis afectan e influyen en los diferentes roles del núcleo familiar (Burgos et al. 2000).

Por otro lado existe una limitación en el tiempo que tiene el profesional de enfermería para la atención de la familia al interior de la unidad, estas limitaciones de tiempo deberían ser un elemento fundamental de análisis que le compete en primer lugar a las directivas institucionales y a la vez a los profesionales de enfermería generando evidencia que permita un ajuste en la carga laboral en la unidades que permita un mayor contacto enfermera-familia-paciente y así conseguir una mayor satisfacción de la familia, impactando de manera positiva en el cuidado, aspectos que se constituyen como la base y sustento de una política menos restrictiva.

De forma adicional las creencias y actitudes de los profesionales de la salud se convierten en otro factor responsable en el tipo de visita, argumentando que los familiares pueden generar interrupciones en las rondas de trabajo, los riesgo de agresión física y emocional por visitantes difíciles, la falta de reconocimiento de las necesidades de los pacientes para la recuperación, las inconsistencias entre las enfermeras en la configuración de los límites con los visitantes, dificultad en el manejo de las necesidades emocionales del paciente y la familia, la presión de los familiares por no dejar la cabecera de la cama del paciente debido a la falta de visitas establecidas y las dificultades para atender las necesidades físicas del paciente y la familia, son las múltiples barreras identificadas por los diferentes autores para la liberalización de la política de visitas en las unidades de cuidados intensivos (Fumagalli et al. 2006).

Por otra parte se considera que la experiencia personal de la enfermera como paciente en estado crítico, la edad, el género y la experiencia laboral en $\mathrm{UCI}$ son variables que influyen en la actitud del profesional de enfermería hacia la visita abierta (Lee et al. 2007).

La restricción de las visitas si bien depende en gran medida de los profesionales de la salud, de manera particular en lo que respecta a las visitas de los niños a las unidades de cuidados intensivos, los familiares juegan un papel importante porque ellos consideran que los niños no son lo suficientemente maduros para entender la situación de su familiar hospitalizado, y por ello el ingreso a la $\mathrm{UCl}$ les podría ocasionar traumas o impactos emocionales, de manera que los familiares opinan que es mejor esperar a que el paciente se recupere y que el niño lo recuerde como antes de su admisión a la UCI (Clarke 2000; Vint 2005).

\section{EFECTOS POSITIVOS DE LAS VISITAS ABIERTAS EN EL PACIENTE CRÍTICO}

Aunque aún existen dudas y preconceptos, diversos estudios han demostrado que los pacientes hospitalizados en unidades de cuidado intensivo y sus familiares obtienen grandes beneficios a nivel físico, emocional y social sobre todo cuando las políticas de visitas son más flexibles y permiten un mayor contacto entre el paciente y su familia.

\section{Aspecto físico}

En la dimensión física se han encontrado cambios de las constantes vitales de los pacientes críticos como aumento de la frecuencia cardiaca y la presión arterial y disminución de la presión intracraneana (PIC) (Livesay et al. 2005), permitiendo una reducción en las complicaciones cardiovasculares y así mismo una disminución de la dependencia física del paciente promoviendo su participación e integración del cuidado con su familia (Vint 2005).

\section{Aspecto emocional}

En la dimensión emocional se ha evidenciado una disminución de la ansiedad y temor en el paciente y los familiares, estos sentimientos son derivados de la oportunidad de información, contacto y comunicación que se establece en el tiempo de las visitas (Vint 2005) así como un beneficio agregado dirigido al paciente critico donde se logra una disminución del stress, percepción de mayor tranquilidad, calma y menor depresión cuando es visitado con mayor frecuencia por su familia (Engström \& Söderberg 2005; Marco et al. 2006), lo cual se explica por la inseguridad y la inestabilidad que surge frente a la posibilidad de muerte de un integrante del grupo familiar. 


\section{Aspecto social}

En la dimensión social se favorece una adecuada relación Familiapaciente-enfermera, lo que promueve una mayor comunicación e interacción con la familia y permite la planificación de un cuidado más individualizado para las personas críticamente enfermas (Achury \& Ramírez 2009).

Sumado a los efectos que generan las visitas en el paciente y en la familia se ha evidenciado que también producen efectos positivos en el profesional de enfermería y su equipo de trabajo incrementando la satisfacción laboral de estos debido a la retroalimentación positiva por parte de los familiares de los pacientes (González et al. 2004; Eriksson \& Bergbom 2007; Kleinnpell 2008).

\section{EVIDENCIA DE ESTUDIOS}

Con el análisis que se ha venido realizando del tipo de visitas, efectos $y$ factores que influyen se pretende agrupar algunos hallazgos relevantes de diferentes estudios que permiten mostrar los efectos positivos de la política de las visitas abiertas en la $\mathrm{UCI}$ tanto en el paciente como en el familiar y la enfermera. (Ver tabla 1 y 2 ).

Es importante destacar la existencia suficiente evidencia que muestra que las visitas restrictivas no son las más adecuadas por los efectos negativos que estas ocasionan en el paciente y la familia; pero aun es necesario seguir realizando estudios en especial de diseño experimental que proporcionen una mayor evidencia sobre la política no restrictivas de las visitas.

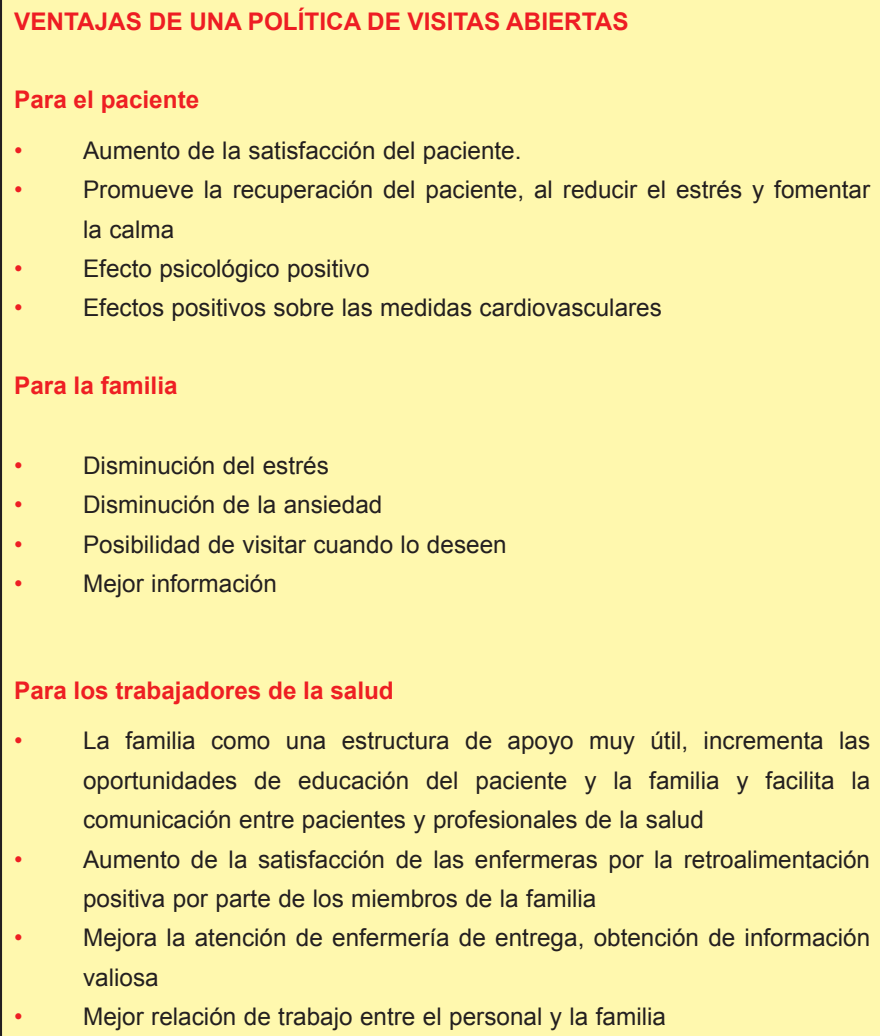

Para los trabajadores de la salud

Tabla 1. Ventajas de una política de visitas abierta (Berti et al. 2007)

\begin{tabular}{|c|c|}
\hline & PERCEPCIONES POSITIVAS \\
\hline ENFERMERA & $\begin{array}{l}\text { - Las enfermeras están de acuerdo en que las visitas } \\
\text { tienen un efecto benéfico en los pacientes y disminuyen } \\
\text { la ansiedad en la familia (Roland et al. 2001; Livesay et } \\
\text { al. 2005; Marco et al. 2006) } \\
\text { La familia representa un apoyo emocional para el } \\
\text { paciente (Livesay et al 2005) (Marco et al 2006) } \\
\text { Las visitas permiten que la familia proporcione } \\
\text { información sobre el paciente y a la vez que la } \\
\text { enfermera conozca las necesidades del paciente y su } \\
\text { familia (Engström \& Söderberg 2005; Marco et al. 2006; } \\
\text { Sims \& Miracle 2006) }\end{array}$ \\
\hline PACIENTE & $\begin{array}{l}\text { Disminuye las complicaciones cardiocirculatorias, la } \\
\text { mortalidad y la ansiedad del paciente hospitalizado } \\
\text { (Henneman \& Cardín 2002) (Sims \& Miracle 2006) } \\
\text { (Fumagalli et al 2006) (Santana et al 2007) } \\
\text { Los pacientes se sienten más tranquilos con la visita } \\
\text { de los familiares (Ramos \& Martínez 2004) (Clarke \& } \\
\text { Harrison 2001) (Bernat 2008) }\end{array}$ \\
\hline FAMILIA & $\begin{array}{l}\text { Los familiares consideran que la apertura de las visitas } \\
\text { permite conocer y comunicarse mejor con el personal } \\
\text { de salud y ello incrementa la satisfacción (Zaforteza et } \\
\text { al 2004; Lee et al. 2007; Knutsson \& Bergbom 2007; } \\
\text { Santana et al. 2007; Pang \& Suen 2009) } \\
\text { Se facilita la comunicación con el personal de salud y } \\
\text { ello permite obtener mayor información sobre el estado } \\
\text { de salud del paciente (Sims \& Miracle 2006; Kutash } \\
\text { 2007) } \\
\text { Los familiares consideran adecuado en tiempo de visita } \\
\text { (Ramos \& Martínez 2004; Knutsson y Bergbom 2007; } \\
\text { Stayt 2007; Bernat 2008) } \\
\text { Los familiares consideran que las visitas se deben } \\
\text { adaptar para satisfacer las necesidades del paciente } \\
\text { y la familia (González et al. 2004; Kutash 2007; Pang } \\
\text { \& Suen 2009) } \\
\text { El ingreso de los niños a la UCl favorece el } \\
\text { entendimiento de la situación, integra a los niños a } \\
\text { la situación familiar y reduce en ellos el sentimiento } \\
\text { de sepa ración y abandono (Clarke 2000; Clarke \& } \\
\text { Harrison 2001; Pochard et al. 2005; Stayt 2007) }\end{array}$ \\
\hline
\end{tabular}

Tabla 2. Percepciones positivas de la política de visitas abiertas en la UCI, en la enfermera, el paciente y la familia

\section{PAPEL DE LA ENFERMERA INTENSIVISTA EN LAS VISITAS}

El eje del actuar del profesional de enfermería basado en la formación integral que este tiene, debe entonces, propender por asegurar el bienestar del ser humano sin importar en el ámbito en que se encuentre, por lo cual se debe tener en cuenta que al brindar el cuidado se debe lograr la satisfacción tanto a nivel físico como psico-emocional del paciente y su entorno familiar (Roland et al. 2001).

La enfermera adquiere un papel determinante en la dinamización de las visitas, pero, surgen numerosos interrogantes a la hora de reorientar las políticas con base en las relaciones con los familiares de pacientes críticos. En primer lugar, no constituye una simple intervención más, sino que conlleva todo un cambio de filosofía en 
el equipo asistencial, no siempre fácil de conseguir por los modelos de práctica profesional fuertemente arraigados en los que no existe un enfoque holístico de la atención. De hecho, las normas de visita en las UCls suelen estar justificadas en argumentos no probados por la investigación, más sometidos a creencias o rutinas de los profesionales que a otras cuestiones (Berti et al. 2007). Por otro lado se piensa que las acciones encaminadas a promover la atención a los familiares de los pacientes críticos tendrán mayor probabilidad de éxito si existe una cierta inclinación hacia el cambio conceptual que supone incluir a los familiares en las dinámicas del paciente.

Es por esto que la enfermera especialista, por su formación, tiene una gran labor, como afirma Simón: "la enfermera clínica especialista puede desempeñar un papel importante en la planificación y ejecución de los cambios necesarios a fin de satisfacer las necesidades y disminuir al mínimo las alteraciones del rol que se presentan durante el periodo de hospitalización de un paciente en la unidad de la cuidado intensivo" (Gavaghan 2002); de manera que la enfermera debe abordar de manera conjunta al familiar del paciente en estado crítico, teniendo en cuenta su estado psico emocional; el conocimiento obtenido por la enfermera especialista debe asegurar el aprovechamiento de la visita a fin de incluir al familiar y hacerlo participe activo en el cuidado directo al paciente critico y así permitir la ejecución de acciones inmediatas ante las reacciones y cambios en el estado del paciente durante el contacto e interacción con su familiar visitante, esto finalmente conseguirá afianzar un vinculo familiar mediante la intervención de enfermería; además, el profesional de enfermería debe ser gestor de cambios a nivel institucional fundamentados en evidencia obtenida a través de productos de investigación propios con sus pacientes y familiares de las unidades donde laboran, para fomentar variaciones en la modalidad de visitas con el fin único de favorecer la rápida recuperación del paciente y la menor alteración posible del vinculo familiar, contribuyendo finalmente al fortalecimiento de la esencia del quehacer de enfermería, brindar un cuidado humanizado, integral y de calidad (Simon et al. 1997).

La Sociedad Andaluza de Enfermería de Cuidados Críticos, consciente de la importancia del profesional de enfermería en las visitas, decidió iniciar un proceso de construcción de recomendaciones, basadas en la evidencia, que ayudan a normalizar la variabilidad preponderante en este campo, estas recomendaciones constituye un aval que puede facilitar la transición desde modelos "cerrados" hacia modelos "centrados en la familia" (Pochard et al. 2005; Velasco Bueno et al. 2007) proporcionando a los profesionales argumentos y fundamentos para situaciones de incertidumbre. A su vez, abren nuevas vías de investigación de cuestiones sin resolver o que precisan resultados de diseños más sólidos que los hasta ahora realizados. A continuación se describen estas conceptualizaciones (Torres 2004; Anzoletti et al. 2008). (Ver Tabla 3.)

\section{CONCLUSIONES}

Durante la estancia de un paciente en una Unidad de Cuidados Intensivos, sus familiares pueden experimentar una serie de sentimientos tales como: estrés, ansiedad, desorganización, desamparo, confusión, preocupación, irritabilidad, culpa, frustración, depresión, incertidumbre, etc., de manera que la necesidad considerada como más importante es la información, la cual debe ser adecuada, honesta, y al mismo tiempo carente de inconsistencias o contradicciones por parte de diferentes miembros del equipo de cuidados de la UCl (Henneman \& Cardin 2002; Garrouste-Orgeas et al. 2008).

La política restrictiva de visitas a la unidad de cuidados intensivos, aumenta el riesgo de enfermedades cardiovasculares en el paciente así como favorece el incremento de los niveles de ansiedad, intranquilidad y desamparo debido a distancia impuesta con sus familiares, de manera que son evidentes los efectos negativos que dicha política de visitas trae consigo y la repercusión sobre el estado de salud del paciente critico. De la misma forma ejerce efectos negativos sobre los familiares de los pacientes críticos debido a que la distancia con sus parientes incrementa los niveles de ansiedad y depresión por el hecho que supone la internación en la unidad de cuidado intensivo; de la misma manera los familiares perciben un mayor grado de insatisfacción de sus necesidades como parientes de pacientes críticos, debido a que se ve reducido el tiempo para la interacción con el personal de salud, la obtención de información sobre el estado de salud, pronostico y tratamiento del paciente y el contacto con su familiar (Vint 2005).La política de visitas no restrictivas en las unidades de cuidados intensivos ejerce efectos positivos sobre todos los actores del proceso: familia- pacienteenfermera. El paciente experimenta una notable reducción en los niveles de ansiedad y con ello la disminución de la incidencia de las complicaciones cardiocirculatorias y la mortalidad; los pacientes críticos se sienten más tranquilos con la presencia constante del familiar ya que este les proporciona una sensación de protección cuando el paciente se siente impotente. En la familia se permite un incremento en la satisfacción de sus necesidades como pariente del paciente crítico debido a que tiene una mayor posibilidad de conocer y comunicarse con el personal de salud, obteniendo así una mayor cantidad y calidad de información sobre el estado de salud del paciente; además la permanencia constante del familiar permite la implicación y participación de este en el cuidado del paciente. $Y$ en el personal de enfermería dicha política de visitas permite un incremento de su satisfacción laborar debido a la retroalimentación positiva por parte del familiar del paciente crítico, además, la mayor interacción con los familiares de la persona hospitalizada le permite obtener una mayor cantidad de información sobre el paciente, de manera que ello le facilita individualización del cuidado (Livesay et al. 2005; Sims \& Miracle 2006; Berti et al. 2007; Kutash 2007).

Existen múltiples factores que influyen en la liberalización o no de las actuales políticas restrictivas de visitas en las unidades de cuidados intensivos; muchos de estos factores se encuentran relacionados con el tipo de unidad y las creencias y percepciones de los familiares y de los profesionales de la salud. Con mayor frecuencia se observa una política liberalizada de vistas en las unidades de cuidados intensivos pediátricos y neonatales al parecer por las evidentes necesidades de este grupo etáreo, además de ello las percepciones y creencias de los profesionales de la salud son otros factores influyentes en la liberalización de visitas, en donde la falta de tiempo para la interacción con el familiar y las posibles falencias en la capacidad de relación y conocimientos, se traducen en temor por parte del personal de salud para la apertura de las vistas en la unidad de cuidados intensivos. De la mima manera los familiares de los pacientes críticos influyen en los aspectos relacionados con el ingreso de los niños a las unidades de cuidado intensivo sustentando su posición en la incapacidad de afrontamiento y entendimiento de la situación que los niños pueden experimentar con el ingreso a la $\mathrm{UCl}$ al visitar a sus parientes en estado crítico (Lee et al. 2007; Aronson et al. 2009).

El profesional de enfermería debe abordar de manera conjunta al familiar del paciente en estado crítico, teniendo en cuenta su estado psico - emocional, el conocimiento obtenido por la enfermera especialista debe asegurar el aprovechamiento de la visita a fin de incluir al familiar y hacerlo participe activo en el cuidado directo al paciente critico y así permitir la toma de acciones inmediatas ante las reacciones y cambios en el estado del paciente durante el contacto e interacción con su familiar visitante; además, el profesional de enfermería debe ser gestor de cambios a nivel institucional fundamentados en evidencia obtenida a través de productos de investigación propios con sus pacientes y familiares de las unidades donde laboran para fomentar variaciones en la modalidad de visitas. 


\section{* La familia y las visitas en las unidades de cuidado intensivo *}

\section{CONCEPTUALIZACION}

- Se entiende por familiar significativo la/s persona/s que mantienen una relación afectiva reconocida por ambos agentes (paciente y familiar), no limitada exclusivamente al vínculo parental.

La PARTICIPACIÓN FAMILIAR es una intervención de carácter progresivo, de manera que la familia puede ir graduando su implicación, a medida que va adquiriendo recursos para ello, tanto internos, como los facilitados por los profesionales sanitarios.

El grado de participación familiar será determinado por el deseo del paciente, el familiar significativo y el equipo asistencial.

En todo paciente ingresado en una Unidad de Cuidados Críticos deberá identificarse a los familiares significativos que potencialmente deseen asumir el rol de cuidadores principales o de soporte.

Entre el/la cuidador/a principal y la enfermera responsable del paciente se establecerá un proceso de comunicación activa y bidireccional que facilite la toma de decisiones compartidas en lo concerniente a los cuidados de su ser querido, negociando los roles de cada uno en todo momento.

El familiar debe tener conocimiento sobre el proceso y la vivencia esperada de la situación, intervenciones, tratamiento, pronostico.

La familia facilita, a su vez, recursos a los miembros del equipo que aumentan las posibilidades de intervención sobre el paciente actualizando de forma permanente con su presencia el rol que desempeñaba el paciente en su familia antes de enfermar, convirtiéndolo en fuente de humanización de los cuidados para los miembros del equipo.

FORMA DE ESCALONAR LA PARTICIPACION DEL FAMILIAR EN EL CUIDADO

- Motivación: toma de conciencia de la posibilidad de ayuda al ser querido mediante la participación en el cuidado y reflexión sobre la operativización de dicha iniciativa (quién, cómo, cuándo,...)

Participación básica: Inicio de la contribución al cuidado en los aspectos que menos demanda, cognitiva y emocional le supongan al cuidador principal. Siempre se individualizarán de acuerdo con las características del paciente y del cuidador.

Participación avanzada: Desarrollo de acciones de ayuda más complejas que requieren una intervención educativa específica.

\section{CARACTERÍSTICAS DE LOS FAMILIRES QUE REQUIEREN ATENCION}

- Diversidad cultural

- Sexo femenino del cuidador

Experiencias anteriores de hospitalizaciones en entornos agresivos

Afrontamiento familiar

Rol del cuidador

Sobrecarga del cuidador familiar

\section{AYUDA EN LA TOMA DE DECISIONES}

- Aquellas decisiones de carácter multidisciplinar en las que se vean implicados más de un profesional, serán informadas y tomadas conjuntamente entre los profesionales afectados y el cuidador/a principal, con la misma metodología de toma de decisiones compartidas.

El proceso de participación familiar en los cuidados debe sustentarse en el establecimiento de un clima de toma de decisiones compartidas entre los agentes implicados: paciente-familiar-enfermera.

Independientemente de la opción elegida por el/la cuidador/a principal, se utilizarán medios de soporte para facilitar la toma de decisiones en los que se incluirá información clara y explícita.

El/la cuidador/a principal sabrá en todo momento cómo y cuándo realizar consultas sobre los cuidados de su ser querido, con la enfermera responsable del paciente o en quien ésta delegue.

COMPETENCIA PROFESIONAL DEL ENFERMERO

La participación en el cuidado como intervención planificada por la enfermera requiere que ésta posea un nivel de competencia adecuado en:

- Entrevista, relación de ayuda.

- Técnicas de comunicación en situaciones difíciles y escucha activa.

- Valoración y diagnóstico de respuestas humanas de afrontamiento, desesperanza, impotencia, déficit de conocimientos, manejo del régimen terapéutico.

Uso de escalas de valoración de función familiar.

ORGANIZACION

- Los Servicios de Salud deben promover activamente la participación familiar en los cuidados bajo un formato metodológicamente validado y con criterios y estándares definidos.

Para poder implementar eficazmente la participación familiar en los cuidados es imprescindible la organización personalizada, mediante asignación de enfermeras responsables de pacientes durante toda la estancia de éste.

Los hospitales deberán articular las medidas organizativas necesarias, de carácter estructural y/o funcional, para que este hecho sea posible de forma sostenida y continuada en el tiempo.

Los hospitales deben facilitar la orientación de los servicios enfermeros orientados hacia la persona, incluyendo la valoración familiar como un aspecto esencial.

Los servicios de Cuidados críticos deberán implantar sistemas de provisión de información a pacientes y familiares con carácter multidisciplinar, estableciendo las medidas oportunas para ello.

MEDIDAS ESPECIFICAS

- No está claro el efecto de la presencia familiar en situaciones de emergencia y se recomienda la investigación en este apartado.

El régimen de visitas deberá adecuarse para que favorezca la dinámica de la participación familiar y será un proceso sujeto a una regulación específica que no interferirá las decisiones que se tomen respecto a la participación familiar en el cuidado.

Se ofrecerá la posibilidad de visitas de menores a sus seres queridos siempre que: se valore previamente el nivel de capacidad del menor, su grado de comprensión de la situación, se le anticipe las características del entorno que va a ver, se produzca en presencia de un progenitor y se evalúe posteriormente el impacto de la visita.

Los Hospitales y Unidades de Cuidados Críticos fomentarán la participación de grupos de apoyo y/o de voluntariado a las familias de pacientes ingresados, que serán coordinados por los miembros del equipo.

Tabla 3. Recomendaciones para la implicación familiar en el cuidado del paciente crítico. [La Sociedad Andaluza de Enfermería de Cuidados Críticos, 2004 (Torres 2004)] 


\section{REFERENCIAS}

Achury L, Ramírez A (2009). Efectos en las enfermeras, familiares y pacientes de la política de visitas abiertas en la UCI. Ene 2009

Anzoletti AB, Buja A, Bortolusso V, Zampieron A (2008). Access to intensive care units: a survey in North-East Italy. Intensive and Critical Care Nursing 24 (6), 366-374.

Aronson PL, Yau J, Helfaer MA, Morrison W (2009). Impact of family presence during pediatric intensive care unit rounds on the family and medical team pediatrics. Pediatrics 124 (4), 1119-1125.

Bernat $\mathrm{R}$ (2008). Necesidades de la familia del paciente crítico: revisión bibliográfica. Nursing 26 (9), 60-63.

Berti D, Ferdinande P, Moons P (2007). Beliefs and attitudes of intensive care nurses toward visits and open visiting policy. Intensive Care Medicine 33 (6), 1060-1065.

Burgos N, Cano P, Garcia C, Lage A, López M (2000). Visitas de familiares a pacientes ingresados en la unidad de cuidados coronarios: opinión de todos los implicados. Enfermería Cardiología VII (20), 28-39.

Clarke CM (2000). Children visiting family and friends on adult intensive care units: the nurses' perspective. Journal of Advanced Nursing 31 (2), 330-338.

Clarke C, Harrison D (2001). The needs of children visiting on adult intensive care units: a review of the literature and recommendations for practice. Journal of Advanced Nursing 32 (1), 61-68.

Díaz Aguila HR, Véliz Sanchez M, Mestre Arceo JR (2006). Encuesta sobre la presencia familiar durante los procederes invasivos y la reanimación. Revista Electrónica de Medicina Intensiva 6 (4), articulo no. A45.

Engström A, Söderberg S (2004).The experiences of partners of critically ill persons in an intensive care unit. Intensive and Critical Care Nursing 20 (5), 299-308.

Engström A, Söderberg S (2005). Close relatives in intensive care from perspective of critical care nurses. Journal of Clinical Nursing 16 (9), 1651-1659.

Eriksson T, Bergbom I (2007). Visits to intensive care unit patients frequency, duration and impact on outcome. Nursing in Critical Care 12 (1), 20-26.

Fernández N (2006). Necesidades de las personas ingresadas en $\mathrm{UCl}$ Metas de Enfermería 9 (5), 19-26

Fumagalli S, Boncinelli L, Lo Nostro A, Valoti P, Baldereschi G, Di Bari M, Ungar A, Baldasseroni S, Geppetti P, Masotti G, Pini R, Marchionni N (2006). Reduced cardiocirculatory complications with unrestrictive visiting policy in an intensive care unit: results from a pilot, randomized trial. Circulation 113 (7), 946-952.

Garrouste-Orgeas M, Philippart F, Timsit J, Diaw F, Willems V, Tabah A, Bretteville G, Verdevainne A, Misset B, Carlet J (2008). Perceptions of a 24-hour visiting policy in the intensive care unit. Critical Care Medicine 36 (1), 30-35.

Gavaghan SR, Carroll DL (2002). Families of critically ill patients and the effect of nursing interventions. Dimensions of Critical Care Nursing 21 (2), 64-71.

Gonzalez CE, Carroll DL, Elliott JS, Fitzgerald PA, Vallent HJ (2004). Visiting preferences of patients in the intensive care unit and in a complex care medical unit. American Journal of Critical Care 13 (3), 194-198.

Henneman E, Cardin S (2002). Family-centered critical care: a practical approach to making it happen. Critical Care Nurse 22 (6), 12-19.

Kleinpell R (2008).Visiting hours in the intensive care unite: more evidence that open visitation is beneficial. Critical Care Medicine 36 (1), 334-335.

Knutsson SE, Bergbom IL (2007). Custodians' viewpoints and experiences from their child's visit to an ill or injured nearest being cared for at an adult intensive care unit. Journal of Clinical Nursing 16 (2), 362-371.

Kutash M (2007). Family members' experiences of the intensive care unit waiting room. Journal of Advanced Nursing 60 (4), 384

Lee MD, Friedenberg AS, Mukpo DH, Conray K, Palmisciano A, Levy MM (2007).Visiting hours policies in New England intensive care unites: strategies for improvement. Critical care Medicine 35 (2), 497501.

Livesay S, Gilliam A, Mokracek M, Sebastian S, Hickey J (2005). Nurses' perceptions of open visiting hours in neuroscience intensive care unit. Journal of Nursing Care Quality 20 (2), 182-189.

Marco L, Bermejillo I, Garayalde N, Satrrate I, Margall M, Asiain M. (2006). Nursing in Critical Care 11 (1), 33-41.

Pang PSK, Suen LKP (2009). Stressors in the intensive care unit: comparing the perceptions of Chinese patients and their family. Stress and Health 25 (2), 151-159.

Pérez M, Rodríguez A, Fernández M, Catalán J, Montejo C (2004). Valoración del grado de satisfacción de los familiares de pacientes ingresados en una unidad de cuidado intensivo. Medicina Intensiva 28 (5), 237-249.

Pochard F, Darmon M, Fassier T, Bollaert PE, Cheval C, Coloigner M, Merouani A, Moulront S, Pigne E, Pingat J, Zahar JR, Schlemmer B, Azoulay E; French FAMIREA study group (2005). Symptoms of anxiety and depression in family members of intensive care unit patients before discharge or death. A prospective multicenter study. Journal of Citical Care 20 (1), 90-96.

Ramos S, Martinez N (2004). Satisfacción familiar en un área intensiva municipal. Revista Cubana de Medicina Intensiva y Urgencias 3 (1), 49-52.

Roland P, Russel J, Richards KC, Sullivan SC, Cox S (2001).Visitation in critical care: processes and outcome of a performance improvement initiative. Journal of Nursing Care Quality 15 (2), 18-26.

Santana L, Sánchez M, Hernández E, García M, Robaina E, Ortiz V (2007). Necesidades de los familiares de pacientes de cuidados intensivos: percepción de los familiares y del profesional. Medicina Intensiva 31(6), 273-280.

Simon S, Phillips K, Badalamenti S, Ohlert J, Krumberger J (1997). Current Practices regarding visitation policies in critical care unit. American Journal of Critical Care 6 (3), 210-217.

Sims J, Miracle V (2006). A look at critical care visitation: the case for flexible visitation. Dimensions of Critical Care Nursing 25 (4), 175180.

Stayt L (2007). Nurses' experiences of caring for families with relatives in intensive care units. Journal of Advanced Nursing 57 (6), 623-630.

Titler M, Walsh S (1992).Visiting critically ill adults. Critical Care Nursing Clinics of North America 4 (4), 623-631.

Torres $P$ (2004). Participación familiar en el cuidado del paciente criticoLa Sociedad Andaluza de Enfermería de Cuidados Críticos, Revista Internacional Para el Cuidado del Paciente Critico 4 (1),1-8.

Velasco Bueno JM (2003).Visita de familiares en UCl: ¿cambio de normas o de cultura? REMI 3 (2), E26 [en Internet].

Verhaeghe S, Defloor T, Van F, Duijnstee M, Grypdonck M (2005). The needs and experiences of family members of adult patients in an intensive care unit: a review of the literature. Journal of Critical Care Nursing 14 (4),501-509.

Vint P (2005). An exploration of the support available to children who may wish to visit a critically adult in ITC. Intensive and Critical Care Nursing 21 (3), 149-159.

Zaforteza C, Gastardo D, Sanchez P, De Pedro J, Lastra P (2004). Relación entre enfermeras de $\mathrm{UCl}$ y familiares: inicios para el cambio. Nure investigación 3 [en Internet]: http://www.nureinvestigacion.es/ ficheros_administrador/original/original3.pdf 